\title{
FORMES DIFFÉRENTIELLES MÉROMORPHES LOCALE- MENT EXACTES. COMPLÉMENTS
}

\author{
PAR \\ PIERRE DOLBEAULT
}

Introduction. Etant donnés une variété analytique complexe $V$ et un ensemble analytique principal $S$ de $V$ sans singularité, désignons par $M_{S}^{p}$ (resp. $m_{S}^{p}$ ) le faisceau des germes de $p$-formes différentielles méromorphes (resp. méromorphes fermées) admettant $S$ comme ensemble polaire à la multiplicité un au plus.

Lorsque $V$ est kählérienne compacte et que l'espace fibré $\{S\}$ défini par $S$ est suffisamment ample au sens de Kodaira et Spencer, on a déterminé, dans un article antérieur [4], la dimension du $q$-ième espace de cohomologie de $V$ à coefficients dans $M_{S}^{p}, m_{S}^{p}$ et $d M_{S}^{p}$ et la dimension d'un espace noté $\mathcal{E}_{S}^{p+1, q}$ déduit des précédents, pour les valeurs de $p$ satisfaisant à certaines inégalités. Ces compléments ont pour but d'achever les calculs pour toutes les valeurs de $p$ et, grâce à [1], sous des hypothèses un peu plus larges. Le Lemme 6, les Propositions 7,13,15, 16 et les Théorèmes 17 et 18 de [4] seront énoncés pour toutes les valeurs de $p$, sous les mêmes numéros et leurs démonstrations seront complétées, en utilisant les mêmes notations.

1. Définition et Lemmes. On supposera désormais que $V$ est une variété kählérienne compacte, de dimension complexe $m$. On imposera à l'ensemble analytique principal $S$ de $V$ les conditions suivantes, ou, au moins, certaines d'entre elles:

(1) $S$ est sans singularité;

(2) la classe caractéristique de l'espace fibré $\{S\}$ est représentable (à l'aide du Théorème de de Rham) par une forme différentielle réelle, de type $(1,1): \gamma=(i / 2 \pi) \sum X_{\alpha \beta^{*}} d z^{\alpha} \wedge d \bar{z}^{\beta}$ telle que la forme $\sum X_{\alpha \beta^{*}} u^{\alpha} \bar{u}^{\beta}$ soit hermitienne, définie positive en chaque point de $V$;

(3) pour tout $p \geqq 0$ et tout $q \geqq 1$, la forme hermitienne

$$
\begin{aligned}
\Theta^{p, q}(\gamma, u ; z)=\sum\left(\delta_{\tau}^{\alpha}\left[X_{\alpha^{*}}^{\beta^{*}}-R_{\alpha^{*}}^{\beta^{*}}\right]+\right. & p R_{\left.\tau \alpha^{*}{ }^{*}\right)}^{\sigma} \\
& \times u_{\sigma \tau_{2} \cdots \tau_{p} \beta^{*} \alpha_{2}^{*}} \cdots_{\alpha q^{*}} \bar{u}^{\tau \tau \tau_{2}} \cdots \tau_{p} \alpha^{*} \alpha_{2}^{*} \cdots \alpha_{q}^{*}
\end{aligned}
$$

dans laquelle $\delta_{\tau}^{\sigma}$ désigne le symbole de Kronecker, $R_{\tau \alpha^{*}}^{\sigma}{ }^{*}$ le tenseur de courbure et $u_{\tau_{1} \cdots \tau_{\alpha_{1}} \ldots \alpha_{q}}$ un tenseur antisymétrique, est définie positive en chaque point $z \in V$.

L'espace fibré $\{S\}$ satisfaisant aux conditions (2) et (3) sera dit suffisamment ample au sens de Kodaira et Spencer $[5 ; 6]$.

On utilisera les théorèmes suivants:

Received by the editors August 2, 1957. 
Lemme A (Akizuki et Nakano). Si $S$ satisfait aux conditions (1) et (2), alors $H^{q}\left(V, \Omega^{p}(\{S\})\right)=0$, pour $p+q \geqq m+1$.

C'est un cas particulier du Théorème 1" de [1].

Lemme B (Kodaira). Si $S$ satisfait aux conditions (1), (2) et (3), alors $H^{q}\left(V, \Omega^{p}(\{S\})\right)=0$, pour $1 \leqq q \leqq m$.

C'est un cas particulier du Théorème 1 de [5].

Lemme C (Akizuki et Nakano). Si $S$ satisfait aux conditions (1) et (2), l'homomorphisme $H^{r}(V, C) \rightarrow H^{r}(S, C)$ induit par l'inclusion de $S$ dans $V$ est un isomorphisme si $r \leqq m-2$, un monomorphisme si $r=m-1$.

C'est un cas particulier du Théorème 2 de [1].

Lemme D. Si $V$ est une variété kählérienne compacte, $S$ un ensemble analytique principal satisfaisant aux conditions (1) et (2), alors,

pour $p+q \geqq m+1$, on $a: H^{p, q}(V, C) \approx H^{m-q, m-p}(V, C)$;

pour $p+q \geqq m$, on $a: H^{p, q}(S, C) \approx H^{m-q-1, m-p-1}(S, C) \approx H^{m-q-1, m-p-1}(V, C)$.

Démonstration. Soit $\mathfrak{H}^{r, s}(V, C)$ l'espace vectoriel des formes harmoniques de type $(r, s)$ sur $V$; il est canoniquement isomorphe à $H^{r, s}(V, C)$. Soit $J$ la forme fondamentale sur $V$. On sait (voir par exemple [2, IX.6.c]) que l'application de toute forme harmonique $\psi^{m-q, m-p}$ sur la forme harmonique $\phi^{p, q}=J^{p+q-m} \wedge \psi^{m-q, m-p}$ définit un isomorphisme de $\mathcal{F}^{m-q, m-p}(V, C)$ sur $\mathcal{F}^{p, q}(V, C)$; il en résulte l'isomorphisme $H^{p, q}(V, C) \approx H^{m-q, m-p}(V, C)$.

L'ensemble analytique principal $S$ est sans singularité (Condition (1)), donc c'est une variété kählérienne compacte admettant pour forme fondamentale $J_{S}$ la forme induite par $J$ sur $S$ et dont la dimension complexe est $m-1$. Si $p+q \geqq m$, on a, d'après la première partie du lemme: $H^{p, q}(S, C)$ $\approx H^{m-q-1, m-p-1}(S, C)$.

De plus: $2 m-p-q-2 \leqq m-2$, alors, d'après le Lemme $\mathrm{C}$, il existe un isomorphisme $H^{2 m-p-q-2}(V, C) \approx H^{2 m-p-q-2}(S, C)$; cet isomorphisme étant induit par l'inclusion, il respecte le type des classes de cohomologie, ce qui établit la dernière assertion du Lemme D.

\section{Résultats auxiliaires.}

Lemme $6^{\prime}$. Si $V$ est une variété kählérienne compacte et si $S$ est un ensemble analytique principal de $V$ satisfaisant aux conditions (1) et (2), alors, l'homomorphisme

$$
a: K^{0, q}(S, C) \rightarrow K^{0, q+2}(V, C)
$$

qui associe, à la classe de cohomologic de toute q-forme fermée $\omega$ sur $S$, la classe de cohomologie du courant $\omega^{\prime}$ sur $V$ défini par

$$
\omega^{\prime}[\psi]=\int_{S} \omega \wedge \psi
$$


possède les propriétés suivantes:

si $q \neq m-1$, c'est un monomorphisme;

si $q=m-1, c^{\prime} e s t$ un épimorphisme.

Démonstration. La démonstration du Lemme 6 de [4] est valable quand on y remplace le Théorème 2 de [6] par le Lemme C; elle établit le Lemme $6^{\prime}$ pour $q<m-3$ et $g \geqq m-1$. Reste à montrer que $a: K^{0, m-i}(S, C) \rightarrow K^{0, m-i+2}(V, C)$ pour $i=2,3$ est un monomorphisme.

On a vu [4] que $a=h^{\prime} \circ j \circ h$, où $h$ et $h^{\prime}$ sont des isomorphismes et $j$ l'homomorphisme $H_{m+i-2}(S, C) \rightarrow H_{m+i-2}(V, C)$ induit par l'inclusion de $S$ dans $V$.

On sait que l'application $\lambda_{S}: \mathfrak{F}^{m-i}(S, C) \rightarrow \mathfrak{H}^{m+i-2}(S, C)$ qui, pour $i=2,3$ envoie toute $(m-i)$-forme harmonique $\phi^{m-i}$ de $S$ sur $J_{S}^{i-1} \wedge \phi^{m-i}$ est un isomorphisme.

Soit $\lambda: \mathfrak{F}^{m-i}(V, C) \rightarrow \mathfrak{H C}^{m+i-2}(V, C)$ l'application qui, pour $i=2,3$, envoie toute $(m-i)$-forme harmonique $\psi^{m-i}$ de $V$ sur $J^{i-1} \wedge \psi^{m-i}$; on sait que c'est un monomorphisme.

On montre comme au (2) de la démonstration du Lemme 6 de [4], que le diagramme suivant est commutatif:

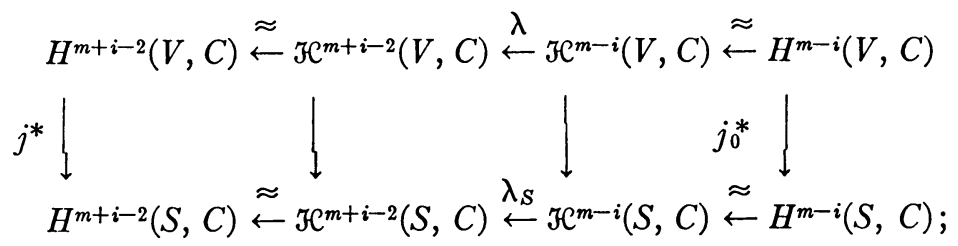

les homomorphismes verticaux sont induits par l'inclusion de $S$ dans $V$, l'homomorphisme $\mathfrak{F C}^{r}(V, C) \rightarrow \mathcal{F}^{r}(S, C)$ appliquant toute $r$-forme harmonique de $V$ sur la composante harmonique de sa restriction à $S$. Il en résulte le diagramme commutatif:

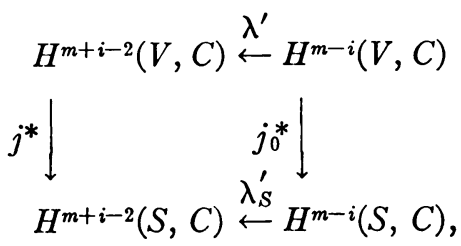

où $\lambda_{S}^{\prime}$ est un isomorphisme et $\lambda^{\prime}$ un monomorphisme. Mais, pour $i=2,3$, d'après le Lemme $C, j_{0}^{*}=\lambda_{S}^{\prime-1} \circ j^{*} \circ \lambda^{\prime}$ est un isomorphisme, donc $j^{*} \circ \lambda^{\prime}$ est un isomorphisme, et $j^{*}$ est un épimorphisme. Il en résulte que $j$, donc $a$, est un monomorphisme, c.q.f.d.

Proposition $7^{\prime}$. Si $V$ est une variêté kählérienne compacte, $S$ un ensemble analytique principal de $V$ satisfaisant aux conditions (1) et (2) alors, pour $p+q \geqq m+1$, on $a$ : 


$$
\operatorname{dim} H^{q}\left(V, \mathcal{R}_{S}^{p}\right)=\operatorname{dim} H^{p-1, q}(S, C)+\operatorname{dim} H^{q}\left(S, \Omega^{p}\left(\{S\}_{S}\right)\right) ;
$$

si, en outre, $S$ satisfait à (3), alors,

pour $p+q \leqq m-2$ ou $p+q=m, q \neq 0$, on $a$ :

$$
\operatorname{dim} H^{q}\left(V, \Re_{S}^{p}\right)=\operatorname{dim} H^{p-1, q}(V, C)+\operatorname{dim} H^{q}\left(S, \Omega^{p}\left(\{S\}_{S}\right)\right) ;
$$

pour $p+q=m-1$, on $a$ :

$$
\begin{aligned}
\operatorname{dim} H^{m-p-1}\left(V, \mathcal{Q}_{S}^{p}\right)= & \operatorname{dim} H^{p-1, m-p-1}(V, C)+\operatorname{dim} H^{p-1, m-p}(V, C) \\
& -\operatorname{dim} H^{p-1, m-p}(S, C)+\operatorname{dim} H^{m-p-1}\left(S, \Omega^{p}\left(\{S\}_{S}\right)\right) ;
\end{aligned}
$$

pour $p=m$ et $q=0$, on $a$ :

$$
\operatorname{dim} H^{0}\left(V, \Omega_{S}^{m}\right)=\operatorname{dim} H^{m-1,0}(S, C) .
$$

Démonstration. (a) Le faisceau $M_{S}^{p}$ étant isomorphe à $\Omega^{p}(\{S\})$, pour $p+q \geqq m+1$ (resp. $1 \leqq q \leqq m$ ), si $S$ satisfait aux conditions (1) et (2) (resp. aux conditions (1), (2) et (3)), d'après le Lemme A (resp. le Lemme B), on a: $H^{q}\left(V, M_{S}^{p}\right)=0$. Alors, on sait [4] que, si $H^{t+1}\left(V, M_{S}^{p}\right)=0$, conoyau $\left[H^{t}\left(V, \Re_{S}^{p}\right) \rightarrow H^{t}\left(V, Q_{S}^{p}\right)\right]$ est isomorphe au noyau de $k^{\prime}: H^{p-1, t+1}(S, C)$ $\rightarrow H^{p, t+2}(V, C)$.

Les parties (b) et (c) de la démonstration de la Proposition 7 de [4] peuvent être complétées ainsi:

(b) On a vu dans [4] que $k^{\prime}$ est la restriction, aux classes de formes de type $(p-1, t+1)$ de l'homomorphisme $a$ du Lemme $6^{\prime}$, après multiplication par $2 \pi i$ et que l'image de $k^{\prime}$ est, au produit près par $2 \pi i$, la partie de l'image de $a$ constituée par les classes de type $(p, t+2)$.

Alors, d'après le Lemme $6^{\prime}$ :

si $p+t \neq m-1, k^{\prime}$ est un monomorphisme, donc le noyau de $k^{\prime}$ est nul et, d'après (a): conoyau $\left[H^{t}\left(V, \Re_{S}^{p}\right) \rightarrow H^{t}\left(V, \Theta_{S}^{p}\right)\right]=0$;

si $p+t=m-1, k^{\prime}$ est un épimorphisme, donc la dimension du noyau de $k^{\prime}$ est:

$$
\begin{aligned}
\operatorname{dim} H^{p-1, m-p}(S, C)-\operatorname{dim} & H^{p, m-p+1}(V, C) \\
& =\operatorname{dim} \text { conoyau }\left[H^{m-p-1}\left(V, \mathcal{Q}_{S}^{p}\right) \rightarrow H^{m-p-1}\left(V, \Theta_{S}^{p}\right)\right] .
\end{aligned}
$$

(c) On a montré, dans [4], que:

$$
\begin{aligned}
\operatorname{dim} H^{q}\left(V, \Re_{S}^{p}\right)= & \operatorname{dim} H^{p-1, q}(S, C)+\operatorname{dim} H^{q}\left(S, \Omega^{p}\left(\{S\}_{S}\right)\right) \\
& -\operatorname{dim} \text { conoyau }\left[H^{q-1}\left(V, \Omega_{S}^{p}\right) \rightarrow H^{q-1}\left(V, \mathcal{Q}_{S}^{p}\right)\right] \\
& -\operatorname{dim} \text { conoyau }\left[H^{q}\left(V, \Omega_{S}^{p}\right) \rightarrow H^{q}\left(V, \Theta_{S}^{p}\right)\right],
\end{aligned}
$$

donc, d'après (b): 
si $p+q-1 \neq m-1, p+q \neq m-1$, on a:

$\operatorname{dim} H^{q}\left(V, \mathbb{R}_{S}^{p}\right)=\operatorname{dim} H^{p-1, q}(S, C)+\operatorname{dim} H^{q}\left(S, \Omega^{p}\left(\{S\}_{S}\right)\right) ;$ en outre, si $p+q \leqq m-2$, on a: $H^{p-1, q}(S, C) \approx H^{p-1, q}(V, C)$, d'après le Lemme D; si $p+q=m-1$, on a:

$$
\begin{aligned}
\operatorname{dim} H^{m-p-1}\left(V, \boldsymbol{Q}_{S}^{p}\right)= & \operatorname{dim} H^{p-1, m-p-1}(V, C)+\operatorname{dim} H^{p-1, m-p}(V, C) \\
& -\operatorname{dim} H^{p-1, m-p}(S, C)+\operatorname{dim} H^{m-p-1}\left(S, \Omega^{p}\left(\{S\}_{S}\right)\right),
\end{aligned}
$$

compte tenu du Lemme D;

si $p+q=m$ et $q \neq 0$, on a:

$$
\operatorname{dim} H^{m-p}\left(V, \mathcal{R}_{S}^{p}\right)=\operatorname{dim} H^{p-1, m-p}(V, C)+\operatorname{dim} H^{m-p}\left(S, \Omega^{p}\left(\{S\}_{S}\right)\right),
$$

compte tenu du Lemme D;

si $p=m$ et $q=0$, on a:

$$
\mathcal{R}_{S}^{m} \approx \Omega^{m-1}[S], \text { donc: } \operatorname{dim} H^{0}\left(V, \Re_{S}^{m}\right)=\operatorname{dim} H^{m-1,0}(S, C) .
$$

Proposition 13'. Si V est une variété kählérienne compacte, $S$ un ensemble analytique principal de $V$ satisfaisant aux conditions (1) et (2) pour $p+t \geqq m$ et aux conditions (1), (2) et (3) pour $p+t<m$, alors on a $k_{1}^{p, t+1}=0$ lorsque $t \geqq 0$.

En effet, dans les conditions de la proposition: $H^{t+1}\left(V, M_{S}^{p}\right)=0$, d'après les Lemmes $\mathrm{A}$ et $\mathrm{B}$ respectivement, donc:

$$
k_{1}^{p, t+1}=\text { conoyau }\left[H^{t}\left(V, \Re_{S}^{p}\right) \rightarrow H^{t+1}\left(V, \Omega^{p}\right)\right]=0 .
$$

Proposition 15'. Si V est une variêté kählérienne compacte, S un ensemble analytique principal de $V$ satisfaisant aux conditions (1) et (2), alors:

pour $p+t \leqq m-1$ ou $p=0, t=m$, on $a$ :

$$
\operatorname{dim} k_{2}^{p, t+1}=\operatorname{dim} K^{p, t+1}(V, C)-\operatorname{dim} K^{p-1, t}(V, C) ;
$$

pour $p+t \geqq m+1$ ou $p>0$, $p+t=m$, on $a$ :

$$
\operatorname{dim} k_{2}^{p, t+1}=0 .
$$

La démonstration de la Proposition 15 de [4] est valable ici, après remplacement du Lemme 6 par le Lemme $6^{\prime}$ et en tenant compte du Lemme D.

Proposition $16^{\prime}$. Si $V$ est une variété kählérienne compacte, $S$ un ensemble analytique principal de $V$ satisfaisant aux conditions (1) et (2) pour $p+t \geqq m$ et aux conditions (1), (2) et (3) pour $p+t<m$, alors:

pour $p>0, \operatorname{dim} k_{3}^{p, t+1}=\operatorname{dim} k_{2}^{p+1, t+1}$, i.e.: 
si $p>0$ et $p+t \geqq m$, on $a: \operatorname{dim} k_{3}^{p, t+1}=0$;

si $p>0$ et $p+t=m-1$, on $a: \operatorname{dim} k_{3}^{p, m-p}=0$;

si $p>0$ et $p+t \leqq m-2$, on $a$ :

$$
\operatorname{dim} k_{3}^{p, t+1}=\operatorname{dim} K^{p+1, t+1}(V, C)-\operatorname{dim} K^{p, t}(V, C) ;
$$

pour $p=0$, on $a: \operatorname{dim} k_{3}^{0, t+1}=\operatorname{dim} K^{1, t+1}(V, C)$.

La démonstration de la Proposition 16 de [4] est valable ici, en y remplaçant la Proposition 15 et le Théorème 1 de [5] par la Proposition 15' et les Lemmes A et B respectivement, et en tenant compte du Lemme D.

\section{Conclusions.}

ThÉORÈME $17^{\prime}$. Si $V$ est une variété kählérienne compacte, $S$ un ensemble analytique principal de $V$ satisfaisant aux conditions (1) et (2), alors:

(A) (a) pour $p+q \geqq m+1$, on $a: I I^{q}\left(V, M_{S}^{p}\right)=0$ et $H^{q}\left(S, \Omega^{p}\left(\{S\}_{S}\right)\right)=0$;

(b) pour $p=0$, on $a: H^{q}\left(V, m_{S}^{0}\right)=H^{q}(V, C)$;

pour $p>0$ et $p+q \geqq m+1$, on $a: \operatorname{dim} H^{q}\left(V, m_{S}^{p}\right)=0$;

pour $p>0, p+q=m$, on $a$ :

$$
\begin{aligned}
\operatorname{dim} H^{m-p}\left(V, m_{S}^{p}\right)= & \operatorname{dim} K^{p, m-p}(V, C)-\operatorname{dim} K^{p-1, m-p-1}(V, C) \\
& -\operatorname{dim} K^{p-1, m-p}(V, C)+\operatorname{dim} K^{p-1, m-p}(S, C) ;
\end{aligned}
$$

pour $p+q \leqq m-1$, on $a$ :

$\operatorname{dim} H^{q}\left(V, m_{S}^{p}\right)=\operatorname{dim} K^{p, q}(V, C)-\operatorname{dim} K^{p-1, q-1}(V, C) ;$

(c) $\operatorname{pour} p>0$ et $p+q \geqq m+1$, on $a$ : $\operatorname{dim} H^{q}\left(V, d M_{S}^{p}\right)=0$;

pour $p=0$ et $q \geqq m+1$, on $a: \operatorname{dim} H^{q}\left(V, d M_{S}^{0}\right)=\operatorname{dim} K^{0, q+1}(V, C)$;

(B) si, en outre, $S$ satisfait à la condition (3), alors:

(a) pour $q>0$, on $a: H^{q}\left(V, M_{S}^{p}\right)=0$; et, de plus:

pour $p+q=m$, on $a: \operatorname{dim} H^{m-p}\left(S, \Omega^{p}\left(\{S\}_{S}\right)\right)=0$;

pour $p+q=m-1$, on $a$ :

$\operatorname{dim} H^{m-p-1}\left(S, \Omega^{p}\left(\{S\}_{S}\right)\right)=\operatorname{dim} H^{p, m-p}(V, C)-\operatorname{dim} H^{p-1, m-p-1}(V, C)$

$$
\text { - } \operatorname{dim} H^{p-1, m-p}(V, C)+\operatorname{dim} H^{p-1, m-p}(S, C) ;
$$

pour $p+q \leqq m-2$, on $a$ :

$\operatorname{dim} H^{q}\left(S, \Omega^{p}\left(\{S\}_{S}\right)\right)=\operatorname{dim} H^{p, q+1}(V, C)-\operatorname{dim} H^{p-1, q}(V, C) ;$

pour $q=0$,

si $p=m$, on $a$ :

$\operatorname{dim} H^{0}\left(V, M_{S}^{m}\right)=\operatorname{dim} H^{m, 0}(V, C)-\operatorname{dim} H^{m-1,0}(V, C)+\operatorname{dim} H^{m-1,0}(S, C) ;$ si $p=m-1$, on $a$ : 
$\operatorname{dim} H^{0}\left(V, M_{S}^{m-1}\right)=\operatorname{dim} H^{m-1,0}(V, C)-\operatorname{dim} H^{m-1,1}(V, C)+\operatorname{dim} H^{m-2,1}(V, C)$

$+\operatorname{dim} H^{m-2,0}(V, C)-\operatorname{dim} H^{m-2,1}(S, C)+\operatorname{dim} H^{0}\left(S, \Omega^{m-1}\left(\{S\}_{S}\right)\right) ;$

si $p \leqq m-2$, on $a$ :

$\operatorname{dim} H^{0}\left(V, M_{S}^{p}\right)=\operatorname{dim} H^{p, 0}(V, C)-\operatorname{dim} H^{p, 1}(V, C)+\operatorname{dim} H^{p-1,0}(V, C)$

$+\operatorname{dim} H^{0}\left(S, \Omega^{p}\left(\{S\}_{S}\right)\right)$;

(b) pour $q>0, p>0$ et $p+q=m$, on a: $\operatorname{dim} H^{m-p}\left(V, d M_{S}^{p}\right)=0$;

pour $q>0, p>0$ et $p+q=m-1$, on $a$ :

$\operatorname{dim} H^{m-p-1}\left(V, d M_{S}^{p}\right)=\operatorname{dim} K^{p, m-p}(V, C)-\operatorname{dim} K^{p-1, m-p-1}(V, C)$

$-\operatorname{dim} K^{p-1, m-p}(V, C)+\operatorname{dim} K^{p-1, m-p}(S, C) ;$

pour $q>0, p>0$ et $p+q \leqq m-2$, on $a$ :

$\operatorname{dim} H^{q}\left(V, d M_{S}^{p}\right)=\operatorname{dim} K^{p, q+1}(V, C)-\operatorname{dim} K^{p-1, q}(V, C) ;$

pour $q>0$ et $p=0$, on $a: \operatorname{dim} H^{q}\left(V, d M_{S}^{0}\right)=\operatorname{dim} K^{0, q+1}(V, C)$;

pour $q=0, p>0$ et $p \neq m-1$, on $a$ :

$\operatorname{dim} H^{0}\left(V, d M_{S}^{p}\right)=\operatorname{dim} K^{p+1,0}(V, C)+\operatorname{dim} H^{0}\left(S, \Omega^{p}\left(\{S\}_{S}\right)\right) ;$

pour $q=0$ et $p=m-1 \neq 0$, on $a$ :

$\operatorname{dim} H^{0}\left(V, d M_{S}^{m-1}\right)=\operatorname{dim} K^{m, 0}(V, C)-\operatorname{dim} K^{m-1,0}(V, C)$

$+\operatorname{dim} K^{m-1,0}(S, C)+\operatorname{dim} H^{0}\left(S, \Omega^{m-1}(\{S\} s)\right) ;$

pour $q=0$ et $p=0$, on $a$ :

$\operatorname{dim} H^{0}\left(V, d M_{S}^{0}\right)=\operatorname{dim} K^{1,0}(V, C)+\operatorname{dim} H^{0}\left(S, \Omega^{0}\left(\{S\}_{S}\right)\right)$.

\section{Démonstration.}

(A) (a) pour $p+q \geqq m+1$, on a: $H^{q}\left(V, M_{S}^{p}\right)=0$ d'après le Lemme A.

Puisque $p+q \geqq m+1$, il suffit de considérer le cas où $q \geqq 1$; alors, d'après la formule (27) de [4] et la Proposition $13^{\prime}$, on a: $\operatorname{dim} H^{q}\left(V, \mathfrak{R}_{S}^{\eta}\right)$ $=\operatorname{dim} H^{p, q+1}(V, C)$, donc, d'après la Proposition $7^{\prime}$ et le Lemme D:

$\operatorname{dim} H^{q}\left(S, \Omega^{p}\left(\{S\}_{S}\right)\right)=\operatorname{dim} H^{p, q+1}(V, C)-\operatorname{dim} H^{p-1, q}(S, C)$

$$
=\operatorname{dim} H^{m-q-1, m-p}(V, C)-\operatorname{dim} H^{m-q-1, m-p}(V, C)=0 .
$$

(b) Pour $p=0$, on a: $m_{S}^{0}=C$, donc $H^{q}\left(V, m_{S}^{0}\right)=H^{q}(V, C)$.

Pour $p>0$, d'après la formule (28) et la Proposition 4 de [4], on a:

$$
\begin{aligned}
\operatorname{dim} H^{q}\left(V, m_{S}^{p}\right)= & \operatorname{dim} K^{p-1, q}(S, C)-\operatorname{dim} K^{p, q+1}(V, C) \\
& +\operatorname{dim} k_{2}^{p, q}+\operatorname{dim} k_{2}^{p, q+1},
\end{aligned}
$$


alors, à l'aide de la Proposition 15', du Théorème 1.14 de [3] et du Lemme D, on obtient les résultats annoncés.

(c) La formule (29) de [4] entraîne:

$$
\begin{aligned}
\operatorname{dim} H^{q}\left(V, d M_{S}^{p}\right)= & \operatorname{dim} H^{q}\left(V, S_{S}^{p}\right)-\operatorname{dim} K^{p+1, q+1}(V, C) \\
& +\operatorname{dim} k_{3}^{p, q}+\operatorname{dim} k_{3}^{p, q+1} ;
\end{aligned}
$$

tenant compte de la Proposition 8 de [4], de la Proposition 16', de (A) (a), du Théorème 1.14 de [3] et du Lemme $\mathrm{D}$, on en déduit la conclusion (A) (c) du théorème.

(B) (a) Pour $q>0$, on a: $H^{q}\left(V, M_{S}^{p}\right)=0$, d'après le Lemme $\mathrm{B}$.

Pour $q>0$, la formule (27) de [4] entraîne:

$$
\operatorname{dim} H^{q}\left(V, \mathbb{R}_{S}^{p}\right)=\operatorname{dim} H^{p, q+1}(V, C)-\operatorname{dim} k_{1}^{p, q}-\operatorname{dim} k_{1}^{p, q+1},
$$

d'où le résultat, d'après les Propositions $7^{\prime}$ et $13^{\prime}$.

Pour $q=0$, la formule (27) de [4] entraîne:

$$
\begin{aligned}
\operatorname{dim} H^{0}\left(V, M_{S}^{p}\right)= & \operatorname{dim} H^{0}\left(V, \Re_{S}^{p}\right)-\operatorname{dim} H^{p, 1}(V, C)+\operatorname{dim} H^{p, 0}(V, C) \\
& +\operatorname{dim} k_{1}^{p, 1} ;
\end{aligned}
$$

compte tenu des Propositions $7^{\prime}$ et $13^{\prime}$ et du Lemme D, cela fournit le résultat annoncé.

(b) La conclusion résulte de la formule (29) de [4], de la Proposition 8 de [4], de la Proposition 16', du Lemme D et du Théorème 1.14 de [3].

THÉOR̀̀me $18^{\prime}$. Soient $V$ une variêté kählérienne compacte et $S$ un ensemble analytique principal de $V$.

Si $q>0$, alors, dans les conditions du Théorème 17':

$$
\operatorname{dim} \varepsilon_{S}^{p+1, q}=\operatorname{dim} H^{q}\left(V, d M_{S}^{p}\right)
$$

a été calculée ci-dessus.

Si S est sans singularité et si $\{S\}$ est suffisamment ample au sens de Kodaira et Spencer, la dimension de l'espace vectoriel des classes de $(p+1)$-formes méromorphes localement exactes dont l'ensemble polaire est $S$ à la multiplicité deux au plus, modulo les différentielles de formes méromorphes sur $V$ dont l'ensemble polaire est $S$ à la multiplicité un au plus est:

pour $p \leqq m-2$ et $p=0$ :

$$
\operatorname{dim} \varepsilon_{S}^{p+1,0}=\operatorname{dim} K^{p, 1}(V, C)-\operatorname{dim} H^{p-1,0}(V, C) ;
$$

pour $p=m-1 \neq 0$ :

$$
\begin{aligned}
\operatorname{dim} \varepsilon_{S}^{m, 0}= & \operatorname{dim} K^{m-1,1}(V, C)-\operatorname{dim} H^{m-2,0}(V, C)-\operatorname{dim} K^{m-2,1}(V, C) \\
& +\operatorname{dim} K^{m-2,1}(S, C) .
\end{aligned}
$$


Démonstration. Pour $q>0$, dans les conditions du Théorème $17^{\prime}$, on a: $H^{q}\left(V, M_{S}^{p}\right)=0$, donc: $\mathcal{E}_{S}^{p+1, q} \approx H^{q}\left(V, d M_{S}^{p}\right)$.

Pour $q=0, p \leqq m-1$, on a:

$$
\operatorname{dim} \mathcal{E}_{S}^{p+1,0}=\operatorname{dim} H^{0}\left(V, m_{S}^{p}\right)-\operatorname{dim} H^{0}\left(V, M_{S}^{p}\right)+\operatorname{dim} H^{0}\left(V, d M_{S}^{p}\right) .
$$

Alors, la conclusion résulte de la Proposition 12 de [4], du Théorème $17^{\prime}$ et du Théorème 1.14 de [3].

\section{BiBLIOGRAPHIE}

1. Y. Akizuki and S. Nakano, Note on Kodaira-Spencer's proof of Lefschetz theorem, Proc. Japan Acad. vol. 30 (1954) pp. 266-272.

1. M. Baldassari, Algebraic varieties, Erg. Math. Berlin, Springer, 1956.

3. P. Dolbeault, Formes différentielles et cohomologie sur une variété analytique complexe, I, Ann. of Math. vol. 64 (1956) pp. 83-130.

4. - Formes différentielles méromorphes localement exactes, Trans. Amer. Math. Soc. vol. 82 (1956) pp. 494-518.

5. K. Kodaira, On a differential-geometric method in the theory of analytic stacks, Proc. Nat. Acad. Sci. U.S.A. vol. 39 (1953) pp. 1268-1273.

6. K. Kodaira and D. C. Spencer, On a theorem of Lefschetz and the lemma of EnriquesSeveri-Zariski, Proc. Nat. Acad. Sci. U.S.A. vol. 39 (1953) pp. 1273-1278.

UNIVERSITY OF BORDEAUX,

Bordeaux, France 